\title{
Modulation of In Vitro Splicing of the Upstream Intron by Modifying an Intra- Exon Sequence Which Is Deleted from the Dystrophin Gene in Dystrophin Kobe
}

\author{
Yasuhiro Takeshima, Hisahide Nishio, ${ }^{*}$ Hiroshi Sakamoto, ${ }^{\ddagger}$ Hajime Nakamura, ${ }^{\star}$ and Masafumi Matsuo \\ Division of Genetics, International Center for Medical Research, and *Department of Pediatrics, Kobe University School of Medicine, \\ Kobe 650, Japan; and ${ }^{\ddagger}$ Department of Biology, Faculty of Science, Kobe University, Kobe 658, Japan
}

\begin{abstract}
Molecular analysis of dystrophin Kobe showed that exon 19 of the dystrophin gene bearing 52-bp deletion was skipped during splicing, although the known consensus sequences at the 5' and 3' splice sites of exon 19 were maintained (Matsuo, M., T. Masumura, H. Nishio, T. Nakajima, Y. Kitoh, T. Takumi, J. Koga, and H. Nakamura. 1991. J. Clin. Invest. 87:2127-2131). These data suggest that the deleted sequence of exon 19 may function as a cis-acting element for exact splicing for the upstream and downstream introns. To investigate this potential role of exon 19 , an in vitro splicing system using artificial dystrophin mRNA precursors (premRNAs) was established. Pre-mRNA containing exon 18, truncated intron 18, and exon 19 was spliced precisely in vitro, whereas splicing of intron 18 was almost completely abolished when the wild-type exon 19 was replaced by the dystrophin Kobe exon 19. Splicing of intron 18 was not fully reactivated when dystrophin Kobe exon 19 was restored to nearly normal length by inserting other sequences into the deleted site. These results suggest that the presence of the exon 19 sequence which is lost in dystrophin Kobe is more critical for splicing of intron 18 than the length of the exon 19 sequence. Characteristically, the efficiency of splicing of this intron seemed to correlate with the presence of polypurine tracks within the downstream exon 19. Moreover, an antisense 31-mer $2^{\prime}$ - $O$-methyl ribonucleotide complementary to the $5^{\prime}$ half of the deleted sequence in dystrophin Kobe exon 19 inhibited splicing of wild-type pre-mRNA in a dose- and time-dependent manner. This first in vitro evidence that dystrophin pre-mRNA splicing can be modulated by an antisense oligonucleotide raises the possibility of a new therapeutic approach for Duchenne muscular dystrophy. (J. Clin. Invest. 1995. 95:515-520.) Key words: exon recognition • exon recognition sequence $\cdot$ minigene $\bullet$ antisense RNA
\end{abstract}

\section{Introduction}

Mutations in the human dystrophin gene are associated with muscular dystrophies of varying degrees of severity, ranging

Address correspondence to Masafumi Matsuo, M.D., Ph.D., Division of Genetics, International Center for Medical Research, Kobe University School of Medicine, 7-5-1 Kusunokicho, Chuo, Kobe 650, Japan. Phone: 78-341-7451; FAX: 78-362-6064.

Received for publication 27 June 1994 and in revised form 30 September 1994

J. Clin. Invest.

(C) The American Society for Clinical Investigation, Inc.

0021-9738/95/02/0515/06 \$2.00

Volume 95, February 1995, 515-520 from the mild Becker (BMD) ${ }^{1}$ form of the illness to the severe Duchenne (DMD) form. DMD is a rapid progressive disease and the affected cases lose the ability to walk independently before the age of $12 \mathrm{yr}$. BMD has a slower rate of progression; affected cases remain ambulatory beyond the age of $16 \mathrm{yr}$ and a few may lead near-normal lives. Large deletions or duplications of the dystrophin gene are present in two thirds of DMD/ BMD cases $(1,2)$. The clinical progression in DMD/BMD patients with deletions can be predicted in $92 \%$ of cases from whether the deletion maintains or disrupts the translational reading frame (frameshift hypothesis) (3-5).

The dystrophin gene consists of 79 exons (6), some of which are alternatively spliced to produce dystrophin isoforms $(7,8)$. Previously we reported three examples of mutation-induced exon skipping dystrophin transcripts (9-11). Others also reported splicing errors due to mutations of the dystrophin gene $(12,13)$. In dystrophin Kobe, a deletion of 52 of the $88 \mathrm{bp}$ in exon 19 results in exon skipping during splicing, even though the known sequences around 5' and $3^{\prime}$ ends of the exon, including the site of lariat formation, are unaffected by the deletion (9). This suggests that a sequence within exon 19 might function as a cis-acting element for correct maturation of dystrophin mRNA.

The cis-acting elements that have been implicated in premRNA splicing are 5' and $3^{\prime}$ splice site sequences, branch point sequences, and their locations (14). These sequences are targets for interactions with trans-acting factors such as small nuclear ribonucleoproteins and other multiple auxiliary proteins. The efficiency of splicing has been shown to be dependent on the length of the downstream exon (15). More recently, several exons have been shown to include purine-rich regions, called exon recognition sequences (ERS), which are necessary for splicing of the upstream intron (16). ERSs are likely targets for splicing factors that identify exon sequences and promote their inclusion in the mature mRNA. Although splicing of pre-mRNA involves accurate splice site selection, recent in vitro studies showed that splicing can be modulated either by altering the exon sequence (16-18) or by changing the binding of heterogeneous nuclear ribonucleoproteins to exon sequence $(19,20)$.

The potential of oligonucleotides to act as modulators of gene expression and as chemotherapeutic agents is currently under intense investigation. The modulation of splicing by antisense oligonucleotide has recently attracted much attention (21, 22). Dominski and Kole (22) recently described an elegant experiment in which aberrant splicing induced by a thalassemia mutation was corrected by an antisense $2^{\prime}-O$-methylribonucleo-

1. Abbreviations used in this paper: BMD, Becker muscular dystrophy; DMD, Duchenne and Becker muscular dystrophy; ERS, exon recognition sequence; 2'-O-Me RNA, 2'-O-methyl ribonucleotide. 


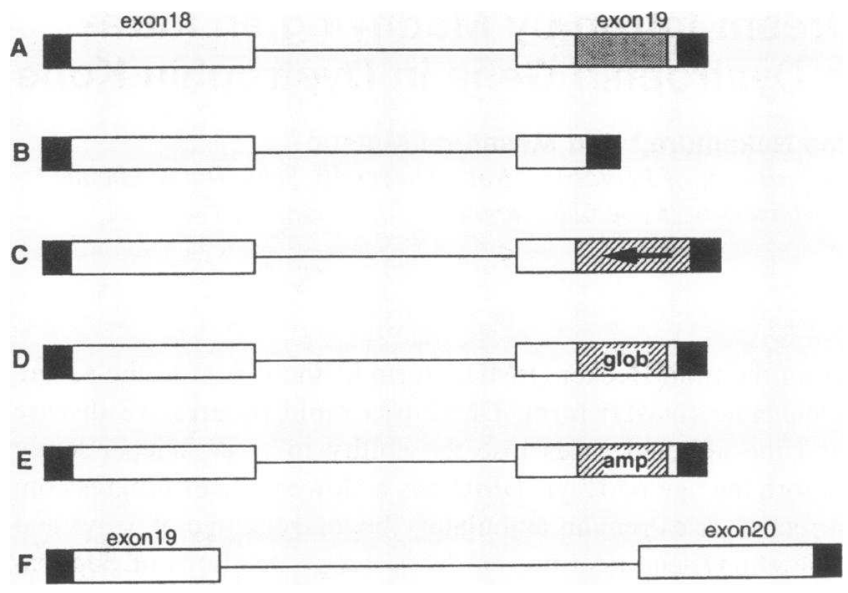

Figure 1. Schematic representation of minigene constructs. The open and filled boxes and lines represent the exons, the vectors, and introns, respectively. In minigene $\mathrm{A}$, sequences of two exons are completely normal, but the size of intron 18 is reduced to $158 \mathrm{bp}$ without altering the general consensus sequences for splicing (for details, see Methods). The shaded area indicates the region that has been deleted in dystrophin Kobe. In minigene B, exon 19 is replaced by that of dystrophin Kobe gene. The deleted region of dystrophin Kobe is reinserted in reverse orientation in minigene $\mathrm{C}$, and portions of $\beta$-globin gene and the ampicillin resistance gene are inserted into the deletion site of minigene $B$ in minigenes $\mathrm{D}$ and $\mathrm{E}$, respectively. The hatched areas indicate the region that has been inserted. The arrow in minigene $\mathrm{C}$ shows the direction of the inserted exon 19 sequence. Glob and amp represent parts of the $\beta$-globin gene and the plasmid pBR322 ampicillin resistance gene, respectively. Minigene F consists of exon 19, truncated intron 19, and exon 20 .

tide ( 2 ' $-O$-Me RNA) . This prompted us to test whether splicing of dystrophin pre-mRNA could also be modulated by an antisense RNA as the first step towards evaluating the potential therapeutic use of antisense RNA to correct aberrant splicing reactions in patients with DMD.

Here we report that splicing of intron 18 of the dystrophin gene is dependent on the sequence of exon 19 and that an antisense $2^{\prime}$ - $O$-Me oligonucleotide complementary to exon 19 sequence can prevent splicing of the upstream intron.

\section{Methods}

Minigene construction. Since intron 18 is very large (23) and thus unsuitable for in vitro experimentation, we reduced its size to $158 \mathrm{bp}$ without altering the consensus splicing site. Minigene $A$ was constructed by ligating two polymerase chain reaction (PCR) products which were amplified from normal genomic DNA by using the methods described previously (24). The ligated product was then inserted into pGEM3Zf(+) (Promega Corp., Madison, WI). This ligated product contains sequences corresponding to exon 18 (99 bp) and the $5^{\prime}$ end of intron 18 (67 bp), the linker sequence ( $3 \mathrm{bp}$ ), the $3^{\prime}$ end of intron 18 (88 bp), and exon 19 (88 bp) (Fig. $1 A$ ). Exon 19 bearing the 52-bp deletion was amplified from dystrophin Kobe genomic DNA and was used to construct minigene B (Fig. 1 B).

To construct minigenes $\mathrm{C}, \mathrm{D}$, and $\mathrm{E}$, three different DNA fragments were inserted into the deleted position of exon 19 of minigene B. In minigene $C$, the deleted sequence in dystrophin Kobe was inserted into minigene $\mathrm{B}$ in reverse orientation (Fig. $1 \mathrm{C}$ ). However, the length of exon 19 was elongated to $97 \mathrm{bp}$ because of the necessity of linker sequence. Minigenes $\mathrm{D}$ and $\mathrm{E}$ have additional sequences derived from exon 2 of the $\beta$-globin gene ( $5^{\prime}$-GGATCCGTTCTTTGAGTCCTTTGGGGATCTGTCCACTCCTGATGCTGTTATG-3') or from the sequence of the plasmid pBR322 ampicillin resistance gene (5'-GGATCCACCACTTCTGCGCTCGGCCCTTCCGGCTGGCTGGTTTATTGCTGAT $-3^{\prime}$ ), respectively (Fig. 1, $D$ and $E$ ). In all three cases, exon 19 was restored to near normal or normal length but the sequence context differed in minigenes $\mathrm{C}, \mathrm{D}$, and $\mathrm{E}$. All minigenes $\mathrm{A}$ to $\mathrm{E}$ were linealized with Sall (New England Biolabs Inc., Beverly, MA). Minigene F, consisting of exon 19 (88 bp), truncated intron 19 (224 bp), and exon 20 ( $99 \mathrm{bp}$ ), was also constructed by ligating PCR products. The fragment was then inserted into pGEM3Zf $(+)$ (Promega Corp.) and it was linealized with HindIII (New England Biolabs Inc.). Sequences of all constructed plasmids were confirmed by automated DNA sequencing (model 373A DNA sequencer; Applied Biosystems, Inc., Foster City, CA) using the Taq dye primer cycle sequencing kit (Applied Biosystems, Inc.)

Preparation of mRNA precursors. Capped pre-mRNAs, which are spliced more efficiently than uncapped RNA (25), were synthesized in vitro as described below. Standard transcription reactions $(25 \mu \mathrm{l})$ contained $2.5 \mu \mathrm{g}$ of each linealized DNA template (minigenes A-F), 40 $\mathrm{mM}$ Tris- $\mathrm{HCl}$ ( $\mathrm{pH} 7.9$ ), $6 \mathrm{mM} \mathrm{MgCl}_{2}, 2 \mathrm{mM}$ spermidine, $10 \mathrm{mM} \mathrm{NaCl}$, $10 \mathrm{mM}$ DTT, $20 \mathrm{U}$ of ribonuclease inhibitor, $500 \mu \mathrm{M}$ each of ATP, CTP, and UTP, $50 \mu \mathrm{M}$ of GTP, $500 \mu \mathrm{M}$ of ${ }^{7 \mathrm{~m}} \mathrm{G}\left(5^{\prime}\right) \mathrm{ppp}\left(5^{\prime}\right) \mathrm{G}, 740$ $\mathrm{kBq}$ of $\left[\alpha-{ }^{32} \mathrm{P}\right] \mathrm{GTP}(15 \mathrm{TBq} / \mathrm{mmol})$, and $20 \mathrm{U}$ of T7 RNA polymerase (Promega Corp.). The mixtures were incubated at $37^{\circ} \mathrm{C}$ for $1 \mathrm{~h}$ followed by phenol/chloroform extraction and ethanol precipitation. Full-length transcripts were purified by denaturing gel electrophoresis as described previously (25).

In vitro splicing. Standard reactions were carried out in a $15-\mu 1$ volume at $30^{\circ} \mathrm{C}$ for the periods indicated. They contained $500 \mu \mathrm{M}$ ATP, $20 \mathrm{mM}$ creatine phosphate, $1.6 \mathrm{mM} \mathrm{MgCl}_{2}, 30 \mathrm{U}$ of ribonuclease inhibitor, $3 \times 10^{4} \mathrm{cpm}$ of pre-mRNA, and $9 \mu \mathrm{l} \mathrm{HeLa} \mathrm{cell} \mathrm{nuclear} \mathrm{extract,}$ which was generously provided by Y. Shimura (Kyoto University, Kyoto, Japan). In some experiments, an indicated amount (1-100 $\mu \mathrm{M})$ of antisense $2^{\prime}$ - $O$-methyl ribonucleotide (2'-O-Me RNA) was added directly to the splicing reaction without any preincubation for annealing. After incubation, reactions were treated with proteinase $\mathrm{K}$, and RNAs were recovered by phenol/chloroform extraction and ethanol precipitation. Splicing products were analyzed by denaturing urea/polyacrylamide gel electrophoresis. Radioactivity of the bands of finally spliced product and mRNA precursors was densitometrically quantitated by using BAS2000 Bio Image Analyzer (Fuji Photo Film Co., Ltd., Kanagawa, Japan). The sizes of the fragments obtained were determined by comparison with end-labeled DNA molecular weight markers produced by MspI digestion of pBR322 (Nippon Gene Co., Tokyo, Japan).

Antisense oligonucleotide. The antisense 2'-O-Me RNA (GCCUGAGCUGAUCUGCUGG CAUCUUGCAGUU) complementary to the first 31 nucleotides of deleted region of the dystrophin Kobe exon 19 was synthesized (Oligos Etc., Inc., Wilengville, OR). The sequence of 31 nucleotides was chosen for this in vitro splicing experiment because these sequences are predicted to form an intra-exon hairpin structure (26).

Identification of spliced products. Mature mRNA produced by in vitro splicing was recovered from the gel and treated with reverse transcriptase as described previously (9). The cDNA thus obtained was amplified by PCR using primers corresponding to segments of exons 18 and 19. Amplified products were subcloned into pT7 Blue T vector (Novagen, Inc., Madison, WI) and sequenced as described above.

\section{Results}

Exon 19 of the dystrophin gene has been reported to be skipped during splicing of pre-mRNA in dystrophin Kobe, which lacks 52 out of 88 nucleotides of exon 19, even though the consensus sequences for splicing are preserved (9). In the previous report 


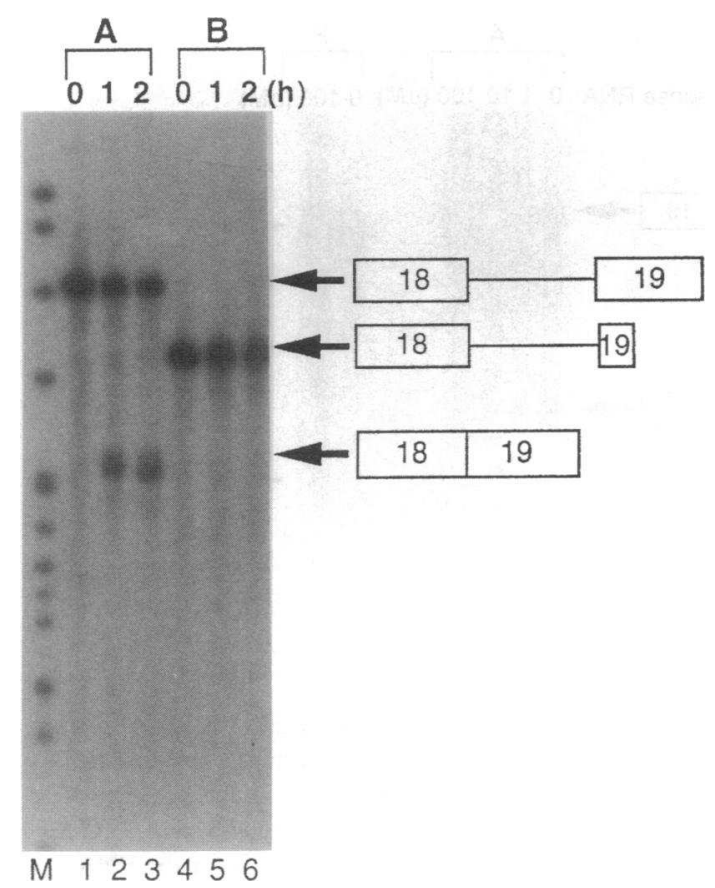

Figure 2. In vitro splicing of dystrophin pre-mRNAs from minigenes $A$ and $B$. Each pre-mRNA from minigene A and B (brackets $A$ and $B$, respectively) was incubated with a $\mathrm{HeLa}$ cell nuclear extract at $30^{\circ} \mathrm{C}$ for the time indicated at the top of each lane. Electrophoresis was carried out using a $5 \%$ polyacrylamide gel containing $8 \mathrm{M}$ urea. The bands for the RNA products are shown schematically at the right side. The molecular size markers are fragments of ${ }^{32} \mathrm{P}$-labeled pBR322 obtained by MspI digestion (lane $M$ ).

we suggested that disappearance of intra-exon hairpin structure is a possible cause of exon skipping in dystrophin Kobe (26). In this report we focused on cis-acting elements and examined the splicing of intron 18 in vitro using five artificial templates in which the internal regions of exon 19 were completely different, as some exon sequences have been shown to function as cis-acting elements for splicing of the preceding intron both in vivo and in vitro $(17,18,27)$. First, essentially wild-type premRNA containing exon 18 , truncated intron 18 , and wild-type exon 19 was synthesized from minigene $A$ (Fig. $1 A$ ) and used as a substrate for in vitro splicing with a HeLa cell nuclear extract. As shown in Fig. 2, this pre-mRNA was efficiently spliced to produce mRNA in which exons 18 and 19 were ligated together (lanes 1-3). The amount of spliced product increased during prolonged incubation. Sequence analysis of this product revealed that exon 18 was precisely joined to exon 19 (data not shown). These results showed that splicing of truncated intron 18 occurred efficiently in this in vitro system.

In contrast, even after $2 \mathrm{~h}$ of incubation we could not detect a band corresponding to the splicing product of pre-mRNA derived from minigene $B$, which bears the same 52-nucleotide deletion within exon 19 as that identified in dystrophin Kobe (Fig. 2, lanes 4-6). The result is consistent with the observation that exon 19 of dystrophin Kobe is skipped in vivo (9) and showed that a portion of exon 19 sequence is necessary for proper splicing of dystrophin pre-mRNA.

There are two possible causes for the splicing deficiency in dystrophin Kobe: the shorter length of exon 19 or the absence

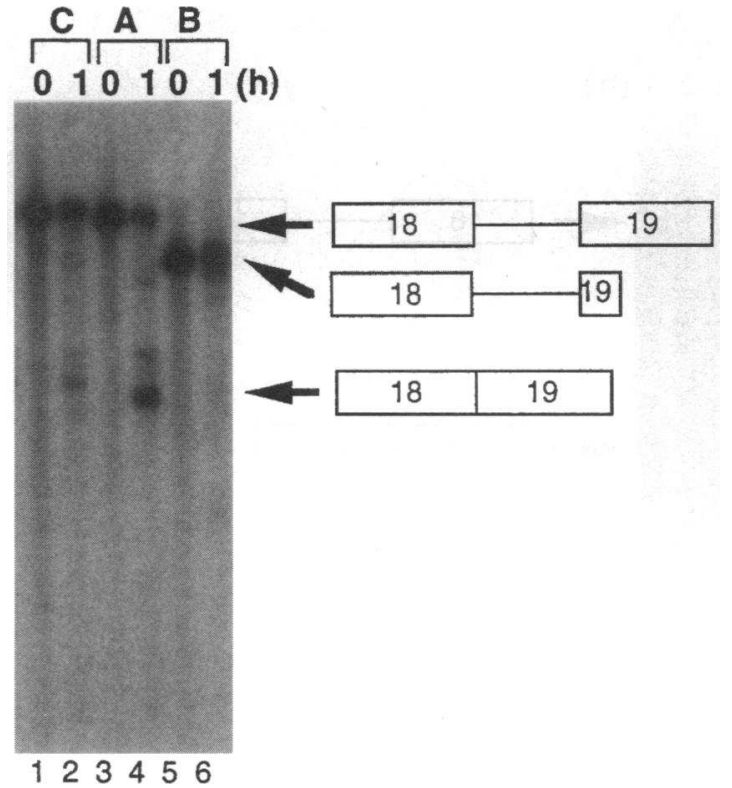

Figure 3. In vitro splicing of dystrophin pre-mRNAs from minigenes A, B, and C. Each pre-mRNA from minigenes A, B, and C ( brackets $A, B$, and $C$, respectively) was incubated with a HeLa cell nuclear extract at $30^{\circ} \mathrm{C}$ for the time indicated at the top of each lane. Lanes 1 and 2: pre-mRNA from minigene $C$; lanes 3 and 4: pre-mRNA from minigene A; lanes 5 and 6: minigene B. Other details are as shown in Fig. 2.

of sequences specifically required for splicing. To distinguish between these possibilities, we examined the in vitro splicing of several pre-mRNAs which were modified versions of minigene $B$ pre-mRNA. Pre-mRNA from minigene $C$, which contains the deleted sequence of exon 19 in reverse orientation, was less efficiently spliced than the wild-type pre-mRNA although the insertion restored the normal length of exon 19 (Fig. 3 , lanes 2 and 4). Densitometric quantification showed that the amount of spliced product from minigene $\mathrm{C}$ pre-mRNA was $\sim 20 \%$ of that from wild-type minigene A pre-mRNA. However, the splicing efficiency of minigene $C$ pre-mRNA was clearly better than that of minigene B pre-mRNA (Fig. 3, lane ๑). This result also indicated that increasing the length of exon could improve splicing efficiency of the upstream intron. In minigenes $\mathrm{D}$ and $\mathrm{E}$, the 52-nucleotide (nt) sequence that is deleted in dystrophin Kobe was replaced by a part of human $\beta$-globin exon $2(52 \mathrm{nt})$ or by a part of a plasmid ampicillin resistance gene (52 nt), respectively (Fig. 1). As shown in Fig. 4 , the splicing efficiency of pre-mRNAs from these constructs was very low compared with wild-type pre-mRNA (minigene D: 9\% [lane 2], minigene E: 3\% [lane 4]). These results clearly showed that the sequence context of exon 19 is quite critical for splicing of intron 18 .

To further examine the role of exon 19 sequence in splicing, we tested the effect of an antisense $2^{\prime}-O$-Me RNA on splicing of wild-type dystrophin pre-mRNA, since antisense $2^{\prime}-O-M e$ RNAs have been shown to anneal efficiently to the target site in the pre-mRNA during splicing and have been used to localize a cis-acting sequence for pre-mRNA splicing (28). When the antisense RNA complementary to the first 31 nucleotides of the deleted 52 nucleotides of dystrophin Kobe was added, splicing 


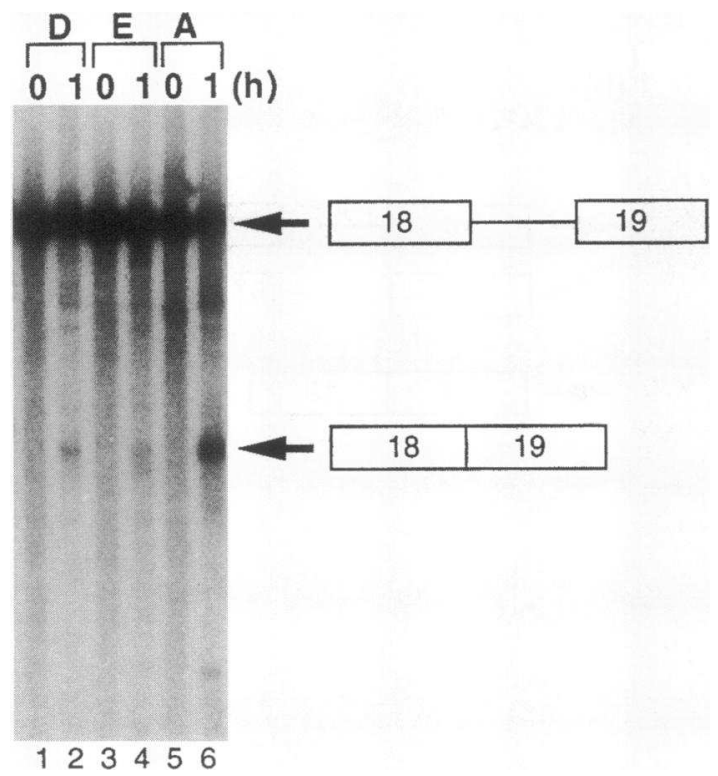

Figure 4. In vitro splicing of dystrophin pre-mRNAs from minigenes $A, D$, and $E$. The effect of the downstream exon sequence context on the preceding intron splicing was examined by replacing deleted region with sequences of a part of the $\beta$-globin gene (minigene $\mathrm{D}$, lanes $I$ and 2 ) or the plasmid ampicillin resistance gene (minigene $\mathrm{E}$, lanes 3 and 4). Other details are as shown in Fig. 2.

of wild-type pre-mRNA from minigene A was drastically inhibited in a dose-dependent manner (Fig. 5, lanes 1-4). In the presence of $1 \mu \mathrm{M}$ of the antisense RNA, the amount of spliced product was reduced to $\sim 20 \%$ of that in its absence. The spliced product was barely detectable in the presence of 100 $\mu \mathrm{M}$ of the antisense RNA.

To exclude the possibility that the antisense RNA inhibits pre-mRNA splicing nonspecifically, we examined its effect on the splicing of another pre-mRNA, which was synthesized from minigene $F$ consisting of exon 19, truncated intron 19, and exon 20 (see Fig. 1). Even $100 \mu \mathrm{M}$ antisense RNA, which drastically reduced splicing of pre-mRNA from minigene $A$, failed to affect splicing of pre-mRNA from minigene F (Fig. 5, lanes 5 and 6 ). Therefore, inhibition by this $2^{\prime}-O$-Me RNA was considered to be highly specific for splicing of intron 18 . These results showed that the first 31 nucleotides of the exon sequence within the deleted region of dystrophin Kobe are necessary for proper splicing.

\section{Discussion}

In this report, we have demonstrated that exon skipping of dystrophin Kobe pre-mRNA is mainly due to the lack of a cisacting exon sequence, rather than to the shorter length of the exon, and that an antisense 31-mer oligonucleotide complementary to exon 19 specifically inhibits splicing of intron 18 . Close inspection reveals that both of the $5^{\prime}$ and $3^{\prime}$ splice sites flanking dystrophin exon 19 are somewhat different from the known consensus sequences (29). For example, the polypyrimidine stretch in the $3^{\prime}$ splice site region of intron 18 is interrupted by a purine residue at position -9 , and the $5^{\prime}$ splice site sequence of intron 19 is also different from the consensus at positions

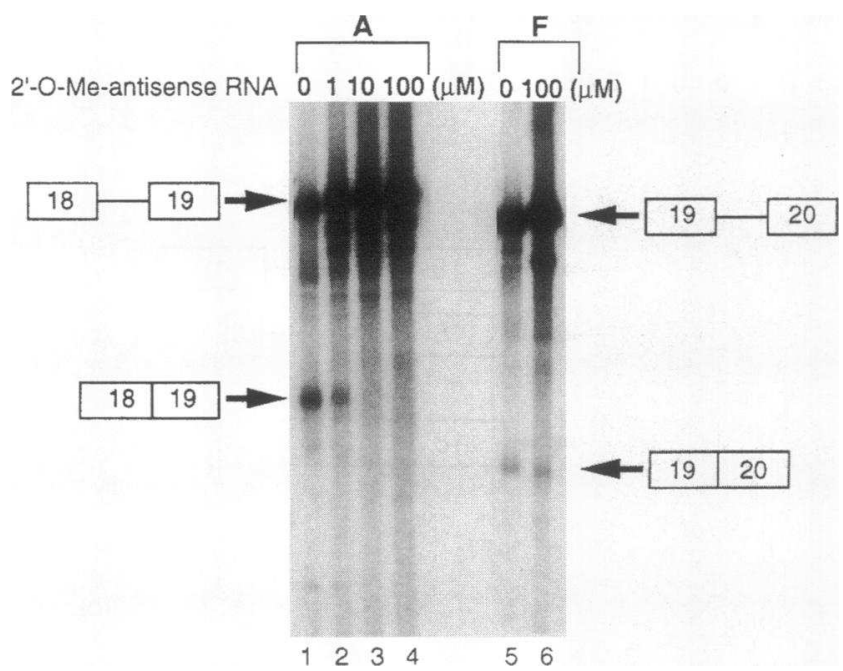

Figure 5. Effect of an antisense 2'-O-Me RNA on dystrophin splicing. In vitro splicing reactions of pre-mRNAs from minigenes A (lanes 1 4) and $\mathrm{F}$ (lanes 5 and $\sigma$ ) were performed for $1 \mathrm{~h}$ in the presence of an antisense $2^{\prime}-O$-Me RNA. The concentration of the antisense 2'-O-Me RNA added is indicated above each lane. The bands for the RNA products are shown schematically at the right and left. Other details are as shown in Fig. 2.

-2 and +5 . In many other cases, such deviations from the consensus are thought to reduce splicing efficiency. It should be noted that the substitution of the consensus $G$ in position +5 is known to produce exon skipping in several systems (30). Thus, other cis-acting element(s) might be needed for proper splicing of intron 19 of normal dystrophin pre-mRNA. Indeed, previous studies suggest that there are two classes of premRNA: those which require specific downstream sequences for splicing and those which do not $(15,31)$. Recently, certain exon sequences, called exon recognition sequences or ERSs, have been shown to function as cis-acting elements required for splicing (16-20). In one particular case, an ERS consisting of polypurine was shown to enhance splicing of the preceding intron (17). Typically, a polypurine stretch with alternating As and Gs can stimulate splicing, while poly (A) or poly (G) cannot. The purine content and the length of polypurine stretch are important for ERS function, and the presence of $\mathrm{U}$ or $\mathrm{C}$ residues within polypurine stretch is deleterious or neutral, respectively. Multiple polypurine stretches act accumulatively.

The results of in vitro studies with synthetic sequence variants of exon 19 reported here show that splicing efficiency is proportional to the number of polypurine stretches in exon 19 (exon 19 of minigenes $C, D$, and $E$ have 2,1 , and 0 polypurine stretches, respectively, see Figs. 3 and 4), consistent with the notion that dystrophin exon 19 needs ERSs for efficient splicing. Indeed, three polypurine stretches which fit the definition of an ERS (GCAAGA, AGCAGA, and GGAACAGA) are located within the 52-nt region that is deleted in dystrophin Kobe. Therefore, it is highly likely that splicing of intron 18 of the dystrophin gene requires cis-activation by polypurine stretches in exon 19. Furthermore, the antisense RNA that inhibited in vitro splicing of intron 18 is complementary to a region of exon 19 which includes two of these three polypurine stretches, suggesting that it acts by preventing ERS function. We realize, 
of course, that the exact mechanism of splicing inhibition by the antisense RNA requires further investigation.

The therapeutic potential of antisense RNA-mediated inhibition of gene activity is exemplified by the inhibition of gene expression in many diseases (32). However, the application of the antisense RNA strategy has been limited by the lack of general rules for designing an efficient antisense RNA system. In addition, all of the experiments described so far used antisense RNAs complementary to mRNA but not to pre-mRNA. However, it has been proposed that targeting nuclear RNA, which is much less abundant than its counterpart in the cytoplasm, may enhance the efficiency of the antisense approach (33).

Antisense $2^{\prime}$ - $O$-Me RNA is shown to form a stable duplex with RNA and to be resistant to RNase and DNase attack (28). Dominski and Kole (22) reported the use of antisense 2 '-O-Me RNA to change pre-mRNA splice site selection. However, the method reported by Dominski and Kole (22) would not be suitable for therapeutic use because the use of RNA complementary to general consensus sequences for splicing would lead to nonspecific splicing inhibition of every pre-mRNA in vivo. The present report describes the second successful attempt to manipulate splicing by antisense $2^{\prime}-O$-Me RNA. Our strategy, which was to target a specific exon sequence, is quite different from that reported by Dominski and Kole (22). This approach circumvents the problem of nonspecific inhibition of splicing and might be developed as a therapeutic tool. The specific inhibition of splicing by antisense RNA might be used to treat certain cases of DMD, in which the consequences of a frameshift deletion could be converted to a more moderate phenotype by changing the splicing pattern so that the reading frame is preserved. For example, a DMD case with a deletion of exon 20 (242-bp long) would be transformed into a BMD phenotype by inducing skipping of exon 19 (88-bp-long). The absence of two consecutive exons should produce an in-frame mutation, resulting in the production of a truncated dystrophin protein with partial dystrophin function. Of course, such a strategy cannot be applied to every deletion mutation of the dystrophin gene, because we do not know which introns require the specific downstream exon sequence for splicing. Many questions must be answered before clinical application: what are the optimal lengths and sequences of antisense oligonucleotides, does the modulation of splicing by antisense RNAs occur in myocytes, and can antisense RNAs be delivered efficiently to muscle nuclei?

In conclusion, we have demonstrated that the 52-bp sequence which is deleted in dystrophin Kobe is required for efficient splicing of the upstream intron, although the possibility that shortening of the exon 19 length is the cause in part of exon skipping could not be totally excluded. The analysis of the exon skipping mechanism reported here leads us to propose that antisense oligonucleotides might be used in DMD therapy.

\section{Acknowledgments}

We thank Dr. Yoshiro Shimura and his colleagues for the generous gift of HeLa cell nuclear extract and Dr. A. Pugsley for advice and critical reading of the manuscript.

This work was supported by grants in aid from the Ministry of Education, Science and Culture of Japan to M. Matsuo and H. Sakamoto, the National Center of Neurology and Psychiatry of the Japanese Minis- try of Health and Welfare, the Nakayama Foundation for Human Science, the Foundation for Therapeutics for Epilepsy to M. Matsuo, Kato Memorial Bioscience Foundation, and Hyogo Science and Technology Association to $\mathrm{H}$. Sakamoto.

\section{References}

1. Koenig, M., E. P. Hoffman, C. J. Bertelson, A. P. Monaco, C. Feener, and L. M. Kunkel. 1987. Complete cloning of the Duchenne muscular dystrophy (DMD) cDNA and preliminary genomic organization of the DMD gene in normal and affected individuals. Cell. 50:509-517.

2. Hoffman, E. P., and L. M. Kunkel. 1989. Dystrophin abnormalities in Duchenne/Becker muscular dystrophy. Neuron. 2:1019-1029.

3. Monaco, A. P., C. Bertelson, S. Liechti-Gallati, H. Moser, and L. Kunkel. 1988. An explanation for the phenotypic differences between patients bearing partial deletions of the DMD locus. Genomics. 2:90-95.

4. Gillard, E. F., J. S. Chamberlain, E. G. Murphy, C. L. Duff, B. Smith, A. H. M. Burghes, M. W. Thompson, J. Sutherland, I. Oss, S. E. Bodrug, et al 1989. Molecular and phenotypic analysis of patients with deletions within the deletion-rich region of the Duchenne muscular dystrophy (DMD) gene. Am. J. Hum. Genet. 45:507-520.

5. Koenig, M., A. H. Beggs, M. Moyer, S. Scherpf, K. Heindrich, T. Bettecken, G. Meng, C. R. Muller, M. Lindlof, H. Kaariainen, et al. 1989. The molecular basis for Duchenne versus Becker muscular dystrophy: correlation of severity with type of deletion. Am. J. Hum. Genet. 45:498-506.

6. Roberts, R. G., A. J. Coffey, M. Borrow, and D. R. Bentley. 1992. Determination of the exon structure of the distal portion of the dystrophin gene by vectorette PCR. Genomics. 13:942-950.

7. Feener, C. A., M. Koenig, and L. M. Kunkel. 1989. Alternative splicing of human dystrophin mRNA generates isoforms at the carboxy terminus. Nature (Lond.). 338:509-511.

8. Ahn, A. H., and L. M. Kunkel. 1993. The structural and functional diversity of dystrophin. Nature Genet. 3:283-291.

9. Matsuo, M., T. Masumura, H. Nishio, T. Nakajima, Y. Kitoh, T. Takumi, J. Koga, and H. Nakamura. 1991. Exon skipping during splicing of dystrophin mRNA precursor due to an intraexon deletion in the dystrophin gene of Duchenne muscular dystrophy Kobe. J. Clin. Invest. 87:2127-2131.

10. Narita, N., H. Nishio, Y. Kitoh, Y. Ishikawa, Y. Ishikawa, R. Minami, H. Nakamura, and M. Matsuo. 1993. Insertion of a $5^{\prime}$ truncated L1 element into the $3^{\prime}$ end of exon 44 of the dystrophin gene resulted in skipping of the exon during splicing in a case of Duchenne muscular dystrophy. J. Clin. Invest. 91:18621867

11. Hagiwara, Y., H. Nishio, Y. Kitoh, Y. Takeshima, N. Narita, H. Wada M. Yokoyama, H. Nakamura, and M. Matsuo. 1994. A novel point mutation $\left(\mathrm{G}^{-1}\right.$ to $\left.\mathrm{T}\right)$ in a $5^{\prime}$ splice donor site of intron 13 of the dystrophin gene results in exon skipping and is responsible for Becker muscular dystrophy. Am. J. Hum. Genet. 54:53-61.

12. Sherratt, T. G., T. Vulliamy, V. Dubowitz, C. A. Sewry, and P. N. Strong. 1993. Exon skipping and translation in patients with frameshift deletions in the dystrophin gene. Am. J. Hum. Genet. 53:1007-1015.

13. Winnard, A. V., Y. Jia-Hsu, R. A. Gibbs, J. R. Mendell, and A. H. M. Burghes. 1992. Identification of a 2 base pair nonsense mutation causing a cryptic splice site in a DMD patient. Hum. Mol. Genet. 1:645-646.

14. Green, M. R. 1986. Pre-mRNA splicing. Ann. Rev. Genet. 20:671-708.

15. Furdon, P. J., and R. Kole. 1988. The length of the downstream exon and the substitution of specific sequences affect pre-mRNA splicing in vitro. Mol. Cell. Biol. 8:860-866.

16. Tanaka, K., A. Watakabe, and Y. Shimura. 1994. Polypurine sequences within a downstream exon function as a splicing enhancer. Mol. Cell. Biol. 14:1347-1354.

17. Watakabe, A., K. Tanaka, and Y. Shimura. 1993. The role of exon sequences in splice site selection. Genes \& Dev. 7:407-418.

18. Caputi, M., G. Casari, S. Guenzi, R. Tagliabue, A. Sidoli, C. A. Melo, and F. E. Baralle. 1994. A novel bipartite splicing enhancer modulates the differential processing of the human fibronectin EDA exon. Nucleic Acids Res. 22:10181022.

19. Sun, Q., A. Mayeda, R. K. Hampson, A. R. Krainer, and F. M. Rottman 1993. General splicing factor SF2/ASF promotes alternative splicing by binding to an exonic splicing enhancer. Genes \& Dev. 7:2598-2608.

20. Mayeda, A., D. M. Helfman, and A. R. Krainer. 1993. Modulation of exon skipping and inclusion by heterogeneous nuclear ribonucleoprotein $\mathrm{A} 1$ and pre-mRNA splicing factor SF2/ASF. Mol. Cell. Biol. 13:2993-3001.

21. Barabino, S. M. L., B. S. Sproat, and A. I. Lamond. 1992. Antisense probes targeted to an internal domain in U2 snRNP specifically inhibit the second step of pre-mRNA splicing. Nucleic Acids Res. 20:4457-4464.

22. Dominski, Z., and R. Kole. 1993. Restoration of correct splicing in thalas- 
semic pre-mRNA by antisense oligonucleotides. Proc. Natl. Acad. Sci. USA. 90:8673-8677.

23. Koenig, M., A. P. Monaco, and L. M. Kunkel. 1988. The complete sequence of dystrophin predicts a rod-shaped cytoskeletal protein. Cell. 53:219228.

24. Matsuo, M., T. Masumura, T. Nakajima, Y. Kitoh, T. Takumi, H. Nishio, J. Koga, and H. Nakamura. 1990. A very small frame-shifting deletion within exon 19 of the Duchenne muscular dystrophy gene. Biochem. Biophys. Res. Commun. 170:963-967.

25. Ohno, M., H. Sakamoto, and Y. Shimura. 1987. Preferential excision of the $5^{\prime}$ proximal intron from mRNA precursors with two introns as mediated by the cap structure. Proc. Natl. Acad. Sci. USA. 84:5187-5191.

26. Matsuo, M., H. Nishio, Y. Kitoh, U. Francke, and H. Nakamura. 1992. Partial deletion of a dystrophin gene leads to exon skipping and to loss of an intra-exon hairpin structure from the predicted mRNA precursor. Biochem. Biophys. Res. Commun. 182:495-500.

27. Steingrimsdottir, H., G. Rowley, G. Dorado, J. Cole, and A. R. Lehmann. 1992. Mutations which alter splicing in the human hypoxanthine-guanine phosphoribosyltransferase. Nucleic Acids Res. 20:1201-1208.
28. Mayeda, A., Y. Hayase, H. Inoue, E. Ohtsuka, and Y. Ohshima. 1990 Surveying cis-acting sequences of pre-mRNA by adding antisense $2^{\prime}-O$-methyl oligonucleotides to a splicing reaction. J. Biochem. 108:399-405.

29. Shapiro, M. B., and P. Senapathy. 1987. RNA splice junctions of different classes of eukaryotes: sequence statics and functional implications in gene expression. Nucleic Acids Res. 15:7155-7174.

30. Krawczak, M., J. Reiss, and D. N. Cooper. 1992. The mutational spectrum of single base-pair substitutions in mRNA splice junctions of human genes: causes and con sequences. Hum. Genet. 90:41-54.

31. Hoshijima, K., K. Inoue, I. Higuchi, H. Sakamoto, and Y. Shimura. 1991 Control of doublesex alternative splicing by transformer and transformer-2 in Drosophila. Science (Wash. DC). 252:833-836.

32. Prockop, D. J., A. Colige, H. Helminen, J. S. Khillan, R. Pereira, and P. Vandenberg. 1993. Mutations in type 1 procollagen that cause osteogenesis imperfecta: effects of the mutations on the assembly of collagen into fibrils, the basis of phenotypic variations, and potential antisense therapies. J. Bone Miner. Res. 8:S489-S492.

33. Volloch, V., B. Schweitzer, and S. Rits. 1991. Inhibition of pre-mRNA splicing by antisense RNA in vitro: effect of RNA containing sequences complementary to exons. Biochem. Biophys. Res. Commun. 179:1593-1599. 